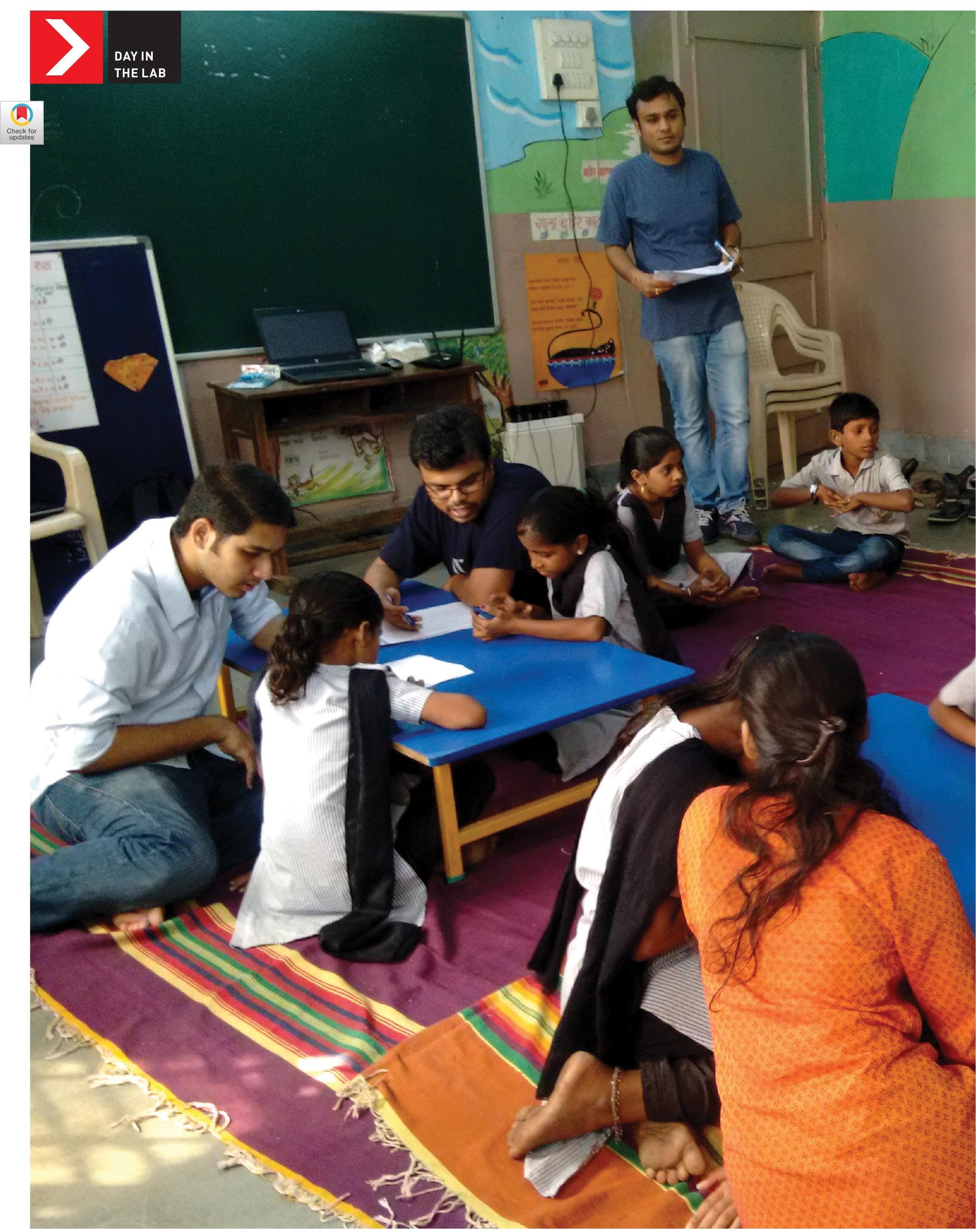




\section{Interaction Design for Indian Needs Lab, IIT Bombay}

As told by Shashank Ahire, Girish Dalvi, Manjiri Joshi, and Anirudha Joshi

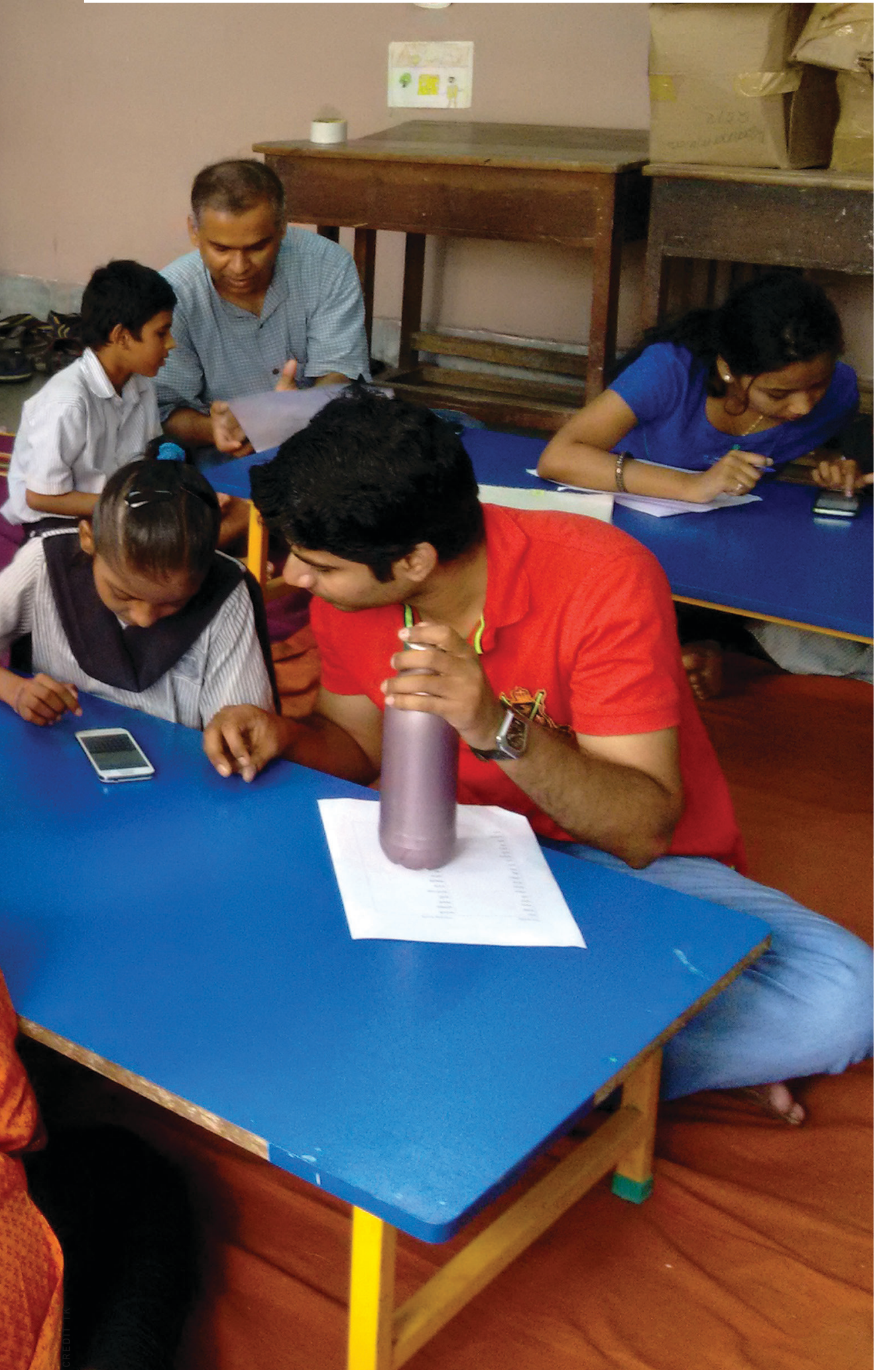

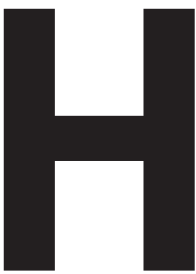

ow do you describe your lab to visitors? We design interactive products to solve the problems of developing countries. We believe that information and communication technologies (ICTs) give us the opportunity to address many problems that have plagued a large number of people in the world for many years.

Our lab is not located in a single room, but rather is made up of people who contribute from different parts of the building, and also from different parts of the country. We are based in a design school, so our focus is on design and the development of products and services. We are one of the few design schools in the world that follow the open source philosophy.

What is a unique feature of your lab? Four things make us unique. First, the domains we work in-literacy, education, text input in complex scripts, and healthcare-have many problems that are particular to developing countries. Second, much of our work happens in the field rather than in the lab. Third, when we start a new project, our focus is on using design to solve problems and creating social impact rather than on producing a research paper. As a result, only a small fraction of our work ever gets published. As it becomes more realistic to use ICTs in the contexts of developing countries, our focus seems to be shifting toward deployment. Fourth, though we use a lot of technology and are located in a sought-after technology university, our group is completely technology agnostic. We tend to use whatever technology fits the context.

How many people are in the lab, and what is the mix of backgrounds and roles? Our lab has a fuzzy boundary. Our more permanent team consists of only eight people, but we keep collaborating with at least 20 other people from within the design school on different projects. Also, we have seven or eight master's students and eight Ph.D. students who work with us. About 10 interns join us every summer. Each year, on average about 35 people are on our team. People come from various backgrounds- 


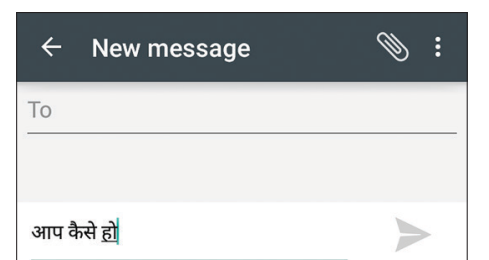

ॐ त्यम्बकं यजामहे सुगन्धिं पुष्टिवर्धनम्। उर्वारुकमिव बन्धनान् मृत्योर्मुक्षीय मामृतात्।

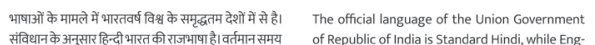
संविधानके अनुसार हिन्दी भारत की राजभाषा है। वर्तमान समय of Republic of India is Standard Hindi, while Engमें कैंद्रीय सरकार में काम हिन्दी और अंग्रेज़ी भाषाओं में होता है, lish is the secondary official language. The constiऔर राज्यों मेंहन्दी अदवा अपनेनअपने क्षेत्राय भाषाओ मे काम tution of india states that "The official language 30 languages are spoken by more than a million native speakers
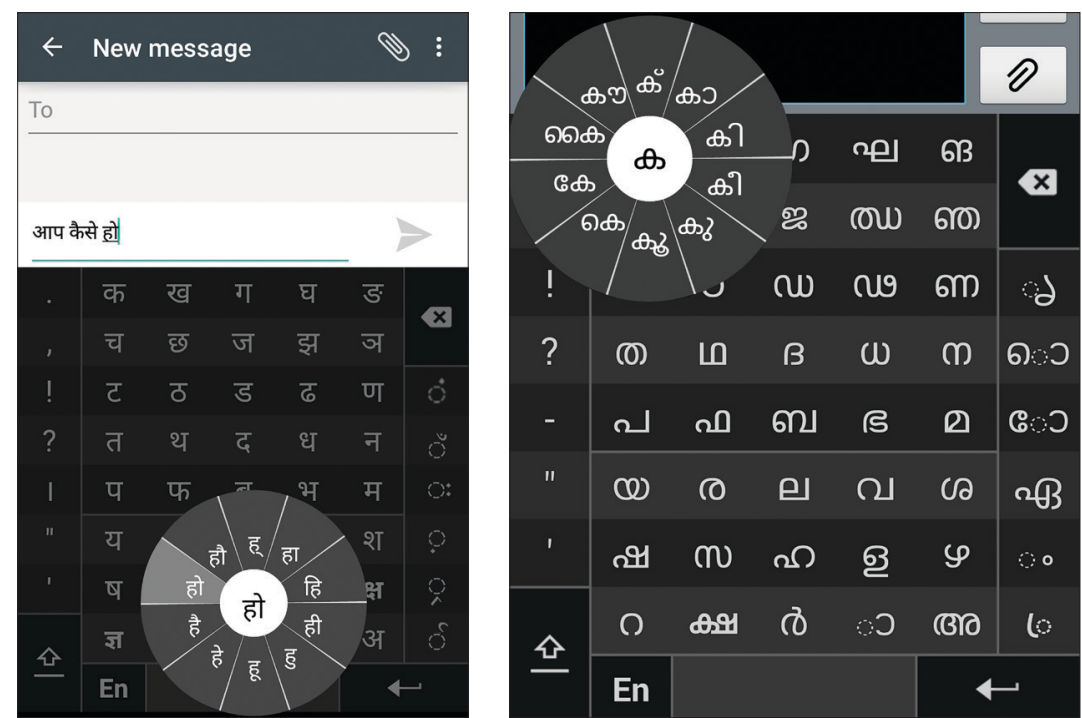

बंकिमचंद्र चटर्जी द्वारा लिखित ‘वंदे मातरम्’

$\rightarrow$ Swarachakra keyboards for Hindi (left) and Malayalam (right).

\section{बंक्मचंद्र चटर्जी द्वारा लिखित ‘वे मातरम्}

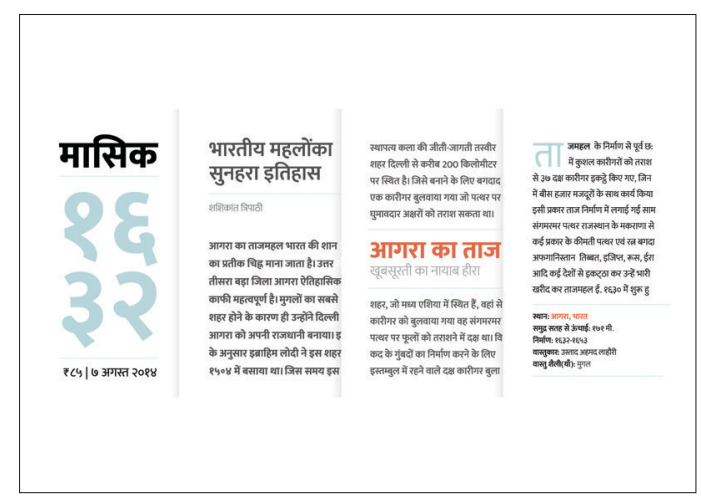

$\rightarrow$ The Ek Mukta

multilingual font.

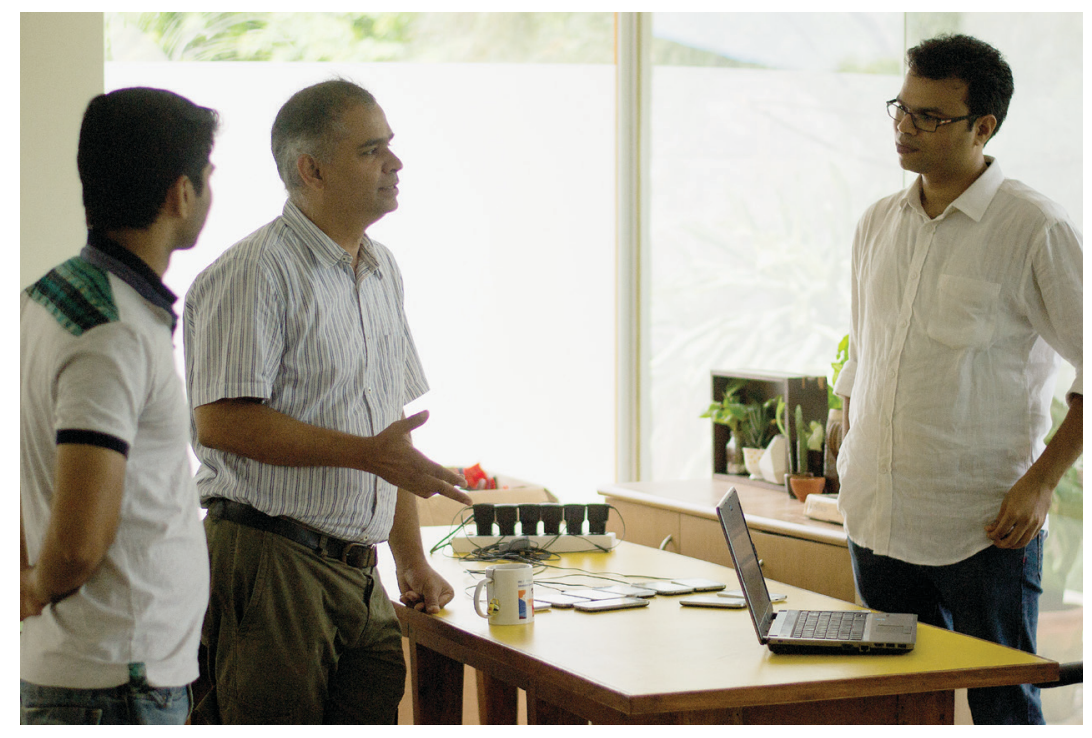

interaction design, computer science, typography, animation, visual communication, product design, filmmaking, writing, psychology.

\section{Briefly describe a day in the life of} your lab. How do people interact? On many days, we are out doing fieldwork, meeting people, making observations, evaluating prototypes, collecting data. Such days are governed by travel schedules, appointments, logistics, and experimental protocols. India is a big country with infrastructure that is barely coping, so this can take up a lot of time and effort.

While in the lab, people do not always have fixed working hours. When the deadline is short and the product has bugs, all-nighters are common. Most ideas come up in impromptu discussions and standup meetings. We juggle multiple projects at the same time, and that keeps us busy. We have regular project presentations, both internal and with people outside. We have paperreading sessions and occasional invited talks. Every project ends with a team dinner.

\section{What projects have you worked} on recently? We have developed Swarachakra, an open source gesture keyboard for touchscreens that we have built for 12 Indian languages so far. The design of Swarachakra is based on the structure of the Indian scripts. In a short period of time and without any commercial promotion, the keyboard has become very popular among users and has also attracted many contributors from across the country. We are also a part of the virtual keyboard standardization consortium for Indian languages, wherein we are developing standardized protocols for evaluating keyboards. In addition, we have developed fonts for Indian languages, as there are few fullfeatured open source fonts. 
$\rightarrow$ Usability testing a keyboard with adults.
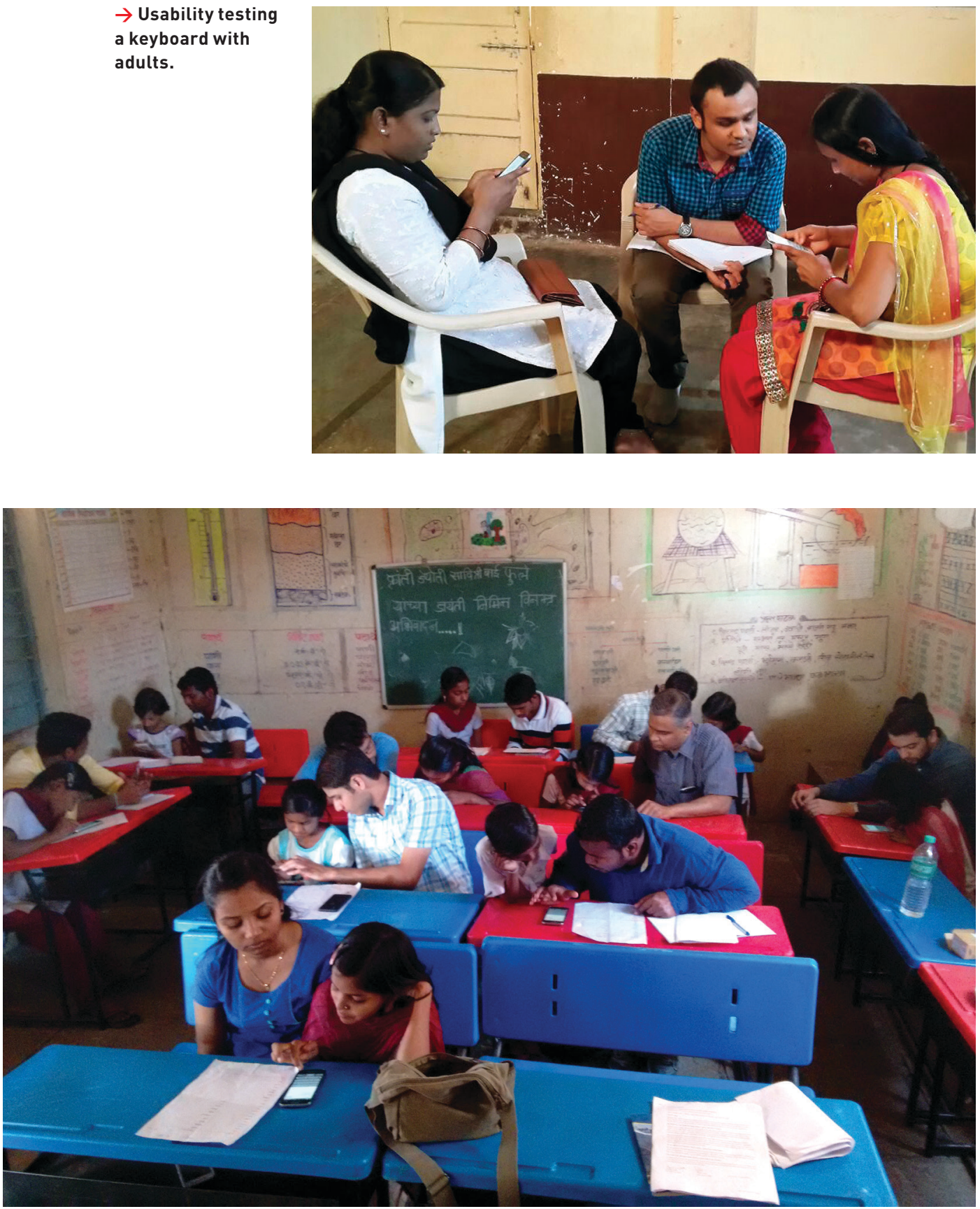

$\rightarrow$ Usability testing a keyboard with children at a school in Nanded, India.
We have been working on a product to help people living with HIV/AIDS manage their medical regimen with phone calls from an interactive voice response system (IVRS). The product is designed to be usable by people with little or no literacy and can reach those who may have only a basic phone.

Besides these implemented projects, we work on several experimental and exploratory design projects.

\section{What is the one thing you see as} most important about the work you do? With rapid adoption of ICTs, on the one hand, we have many opportunities to solve problems of human development. On the other hand, with the equally rapid advent of globalization and corporatization, local cultural identities are threatened. The scope of our work is deliberately local. We are deeply committed to using ICTs to solve the problems that we see in our immediate environment, and we also are passionate about preserving the rich cultural diversity that we have inherited.

http://idid.in/
We have developed fonts for Indian languages, as there are few full-featured open source fonts. 